\title{
Ultrasound guided vacuum assisted excision of breast papillary lesions with no atypia: Single centre experience after 2018 UK Breast Screening guidelines
}

Teresa Presa Abos ${ }^{1 *}$, Jaspal Juttla ${ }^{1}$, Amrita Kumar ${ }^{1}$, Rezan Gardy² and Margaret Moreland ${ }^{1}$

*Correspondence: tpresa@icloud.com

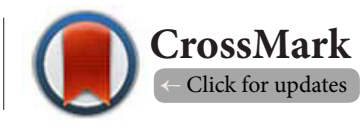

'Consultant Breast Radiologist. The Parapet Breast Screening and Symptomatic Unit. Frimley Health NHS Foundation Trust, UK.

${ }^{2}$ Consultant Breast Pathologist. Frimely Health NHS Foundation Trust, UK.

\begin{abstract}
Purpose: Papillary lesions with no atypia (PLNA) are breast lesions with unknown potential of malignancy (B3). New B3 guidelines published in 2018 by NHS Breast Screening Programme proposed VAE (vacuum assisted excision) of PLNA as an alternative to open surgical excision. This study aims to summarise our results using ultrasound guided VAE as diagnostic and therapeutic tool for PLNA, as well as to demonstrate how the technique works and describe the patient experience.

Material and Methods: Retrospective analysis of the US guided VAE (7G needle) for treatment of PLNA since July 2018. We reviewed the procedure, time taken, technical specifications, potential complications and the results.

Results: Twenty one PLNA were excised. The size of the lesions was 5- $28 \mathrm{~mm}$. Eighteen of the lesions were completely excised. In 16 of the lesions a post biopsy clip was inserted.

The procedure lasted an average of 30 minutes (including consenting and positioning). During and after the procedure there were no complications.

Twenty of the excised lesions came back as papillary lesions with no atypia. One of the lesions was up graded to atypical ductal hyperplasia. None of them were upgraded to B5 lesions.

Conclusion: Ultrasound guided VAE could be used as first line treatment or diagnosis of papillary lesions with no atypia. The procedure is simple and easy to perform with no complications. In the majority of the cases the lesion can easily be removed completely.
\end{abstract}

Keywords: Breast, Papillary lesion, Papilloma, No atypia, VAE, Ultrasound, B3, Upgrade, Excision, Unkown potential of malignancy, Screening

\section{Introduction}

Papillary lesions encompass a variety of lesions, which include papillary lesions with no atypia (papilloma), papillary lesions with atypia, intraductal papilloma with ductal carcinoma in-situ (DCIS), papillary DCIS and papillary carcinoma [1]. Intraductal papilloma with DCIS, papillary DCIS and papillary carcinomas are considered malignant lesions and treated as such. Both papillary lesions with no atypia or with atypia are considered lesions with unknown potential of malignancy (B3 lesion), with a potential to upgrade to a malignant lesion. The percentage of upgrade varies depending on the series from 0-28\% [2], therefore the management of these lesions has been a matter of debate for many years, including surgical excision or follow up. Nevertheless, it is clear that the upgrade to malignancy of lesions with atypia is higher than those without atypia.

In 2018 NHS Breast Screening Programme (NHSBSP) published new guidelines [2] for the management of B3 lesions (Figure 1). The guidelines proposed a second line imaging guided vacuum assisted excision (VAE) of the lesion after an initial diagnosis of papillary lesion with no atypia. For those 
lesions originally diagnosed with atypia, due to the higher potential risk of associated malignancy, the guidelines suggest a surgical excision of the lesion.

Our breast screening and symptomatic unit embraced the new NHSBSP guidelines since August 2018 and every woman diagnosed with a papillary lesion with no atypia is offered a vacuum excision of the lesion under ultrasound (US) guidance. This study aims to summarise our results as well as demonstrate how the technique works and describe the patient experience.

\section{Material and methods}

Since August 2018 to September 2019, 21 patients have been diagnosed with papillary lesion with no atypia. The majority of the patients were diagnosed through the screening programme, but those lesions diagnosed incidentally on a symptomatic clinic were also included. All the 21 patients had a previous US guided core biopsy (14G) of the lesion confirmed histologically as papillary lesion with no atypia and the pathologist classified all of them as B3.

Two papillary lesions with no atypia were excluded from the sampling, as patients' required primary surgical management for symptom control (bloody nipple discharge).

The other twenty one women were verbally consented for the procedure and given an information sheet about the procedure (Appendix 1). Potential complications, including pain during the biopsy, bleeding, infection or nipple or skin damage were discussed with the women on the day of the procedure. All the women agreed to the procedure. A written modified surgical WHO checklist was completed (Appendix 2).

A clean technique was used for the procedure. All lesions were localised with ultrasound. After cleaning the skin with alcoholic solution, 2-5 mls of lidocaine $2 \%$ local anaesthetic was infiltrated subdermally and into the superficial tissues of the needle tract. This was followed by deeper infiltration around thelesion using $5-10 \mathrm{mls}$ of xylocaine $1 \%$ with adrenaline (1:200.000).

After a small scalpel incision in the skin, a 7G needle was inserted below the lesion (EnCor ${ }^{\circ}$ vacuum assisted breast biopsy system, Bard).

After the VAE, a clip was inserted to allow future localisation if needed. A radiology assistant compressed the biopsied area for 5 to 10 minutes after the excision and the area was cleaned and dressed. Standard written post biopsy care was given with a recommendation of no heavy lifting or strenuous exercise for 24 to 48 hours (Appendix 3).

\section{Results}

Twenty one lesions were excised with an US guided VAE. The average size of the lesion was $10 \mathrm{~mm}(5-28 \mathrm{~mm})$. All the lesions were graded on the original US as $\mathrm{U} 2$ or $\mathrm{U} 3$, none of the lesions had suspicious findings under the ultrasound. Eighteen of the lesions were completely sonographically excised. Three of the lesions had residual areas on the ultrasound after the excision, but were widely sampled. The number of cores needed for a thorough sampling of the lesion or complete removal was between 3 cores and 12 cores with an average of 6.8 cores. In 16 of the lesions a clip was introduced at the end of the procedure. In 5 patients a clip was not inserted due to patient choice (in 3 cases) and due to technical issues (Clip did not deploy in 2).

The procedure lasted an average of 30 minutes, including the verbal consent, the localisation of the lesion under the US and the post-biopsy care. During the procedure none of the women complained about pain or discomfort. There was no heavy or unexpected bleeding. The compression of the area after the biopsy lasted about 5-10 minutes.

The final histology showed 16 lesions were papillary lesion with no atypia, and coded E3. In 4 patients, no residual papillary lesion was identified, and benign findings were seen including columnar cell changes, usual ductal hyperplasia and a fibroadenoma. One of the patients was upgrade to atypical findings with atypical ductal hyperplasia. No upgrade to malignancy was found in any of the patients. The percentage of upgrade to malignancy was $0 \%$.

No correlation was found between size of tumour, or ultrasound appearances and upgrade to malignancy or atypia. In 10 of the lesions the pathology report identified a previous scar or inflammation from the initial core biopsy. In the other 11 patients there was no mention to the previous biopsy site. Immunohistochemistry was compatible with benign findings in all the 21 cases (Smooth muscle myosin (SMM), cytokeratin (CK) 14 and CK5/6).

Sixteen women were discharged back to the Screening Programme or discharged completely from the clinic. One patient continued on the Family History Screening Programme as per her recommendation. Two patients continued under annual surveillance due to the presence of multiple papillary lesions and due to the presence of atypia.

\section{Discussion}

Papillary lesions of the breast are a heterogeneous group of lesions consisting of branch-like fronds with fibrovascular cores and overlying lining epithelium [2] (Figure 2). The papillary lesions are relatively frequent lesions in the breast, with some authors recording up to $5 \%$ of all the breast biopsies [3]. The WHO classification of breast tumours differentiate two groups, one with unknown potential of malignancy, classified as B3 (including papillary lesions with or without atypia) and a second group classified as malignant, usually $\mathrm{B} 5 \mathrm{a}$ (including papillary lesion with DCIS, papillary DCIS or papillary carcinoma) (Table 1).

The papillary lesions graded as B3 (with and without atypia), have a wide variety of presentations. They can be found incidentally on a screening mammogram or presented symptomatically as a lump or nipple discharge.

The mammograms may be normal or present as indeterminate lesions, like asymmetric density well defined lesions or clustered microcalcifications. On ultrasound the lesions may have completely benign features or indeterminate features 


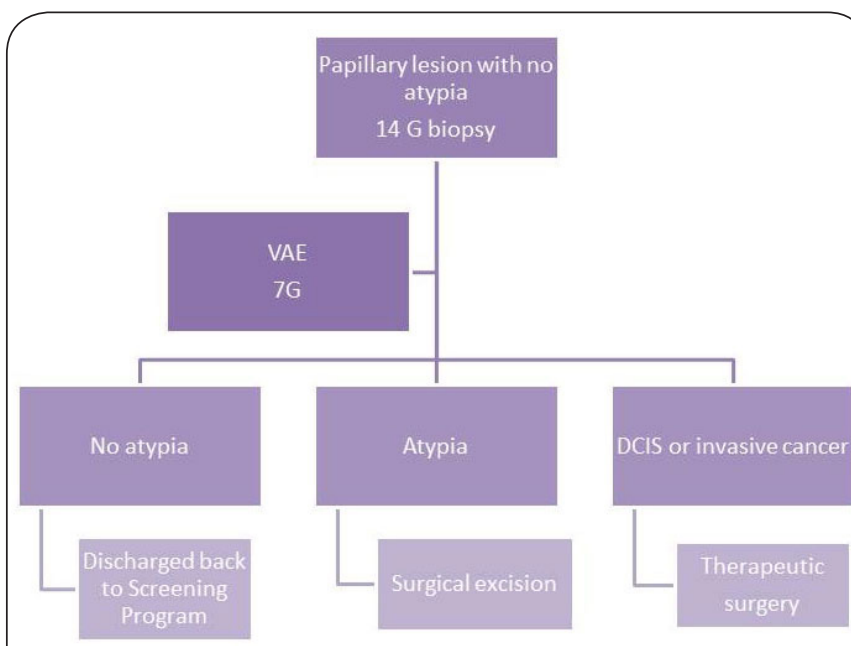

Figure 1. NHSBSP guidelines for managing papillary lesions with no atypia.

Table 1.

\begin{tabular}{|l|l|l|}
\hline Type of lesion & Histological classification & Proposed management \\
\hline Papillary lesions with no atypia & B3 & VAE \\
\hline Papillary lesions with atypia & B3 & Surgical excision \\
\hline $\begin{array}{l}\text { Papillary lesions with DCIS } \\
\text { Papillary DCIS }\end{array}$ & B5a & Surgical treatment \\
\hline $\begin{array}{l}\text { Papillary carcinoma } \\
\text { (encapsulated papillary } \\
\text { carcinoma, solid papillary } \\
\text { carcinoma or invasive papillary } \\
\text { carcinoma) }\end{array}$ & B5a, b, c & Surgical treatment \\
\hline
\end{tabular}

like welldefine masses, rounded or oval or echogenic lesions within a duct (Figures 3-4).

In the majority of the cases the B3 papillary lesions are diagnosed initially with an ultrasound or stereotactic guided biopsy.

The challenge of B3 lesions, including the papillary lesions, is that the lesions have a risk of associated DCIS or malignancy within the B3 lesion. The most important predictor of upgrade to malignancy is the presence of atypia; with a $9-13.2 \%$ upgrade in papillary lesions with no atypia and $36-47.8 \%[4,5]$

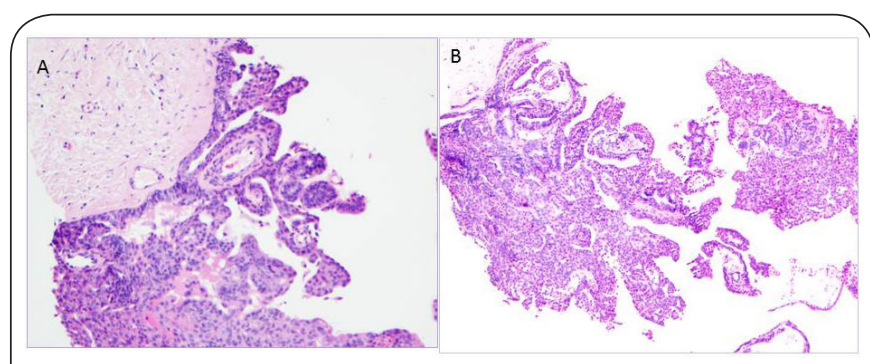

Figure 2. HE of a papillary lesion with no atypia. A. Arborizing fibrovascular papillary lesion with an adjacent normal stroma. B. The papillary lesion showing no atypia.

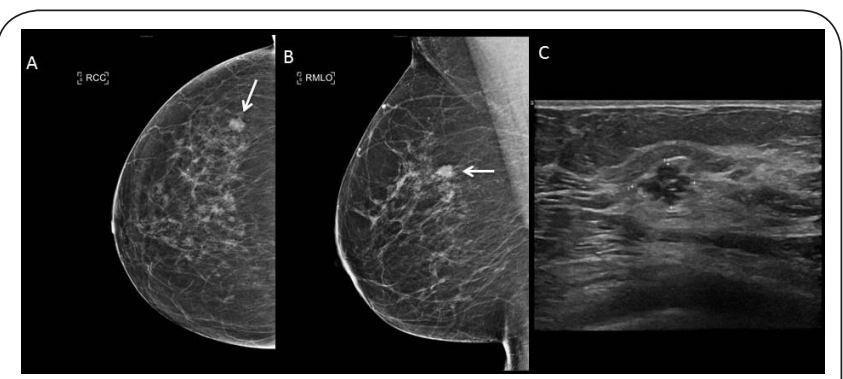

Figure 3. A,B CC and MLO mammographic views showing an irregular lesion in the right upper outer quadrant (arrow). C. US correlation showed an oval lobulated cystic lesion proven as papillary lesion with no atypia in the $14 \mathrm{G}$ core biopsy.

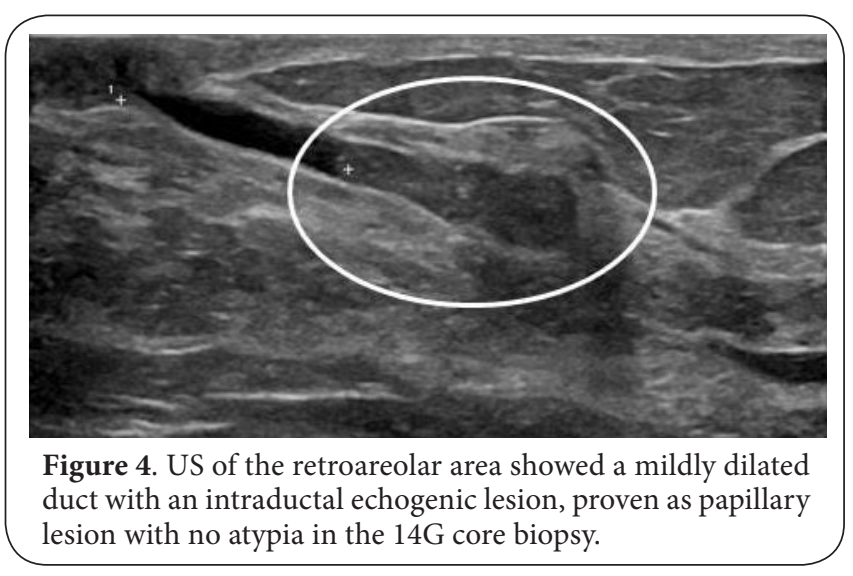

in papillary lesions with atypia. Due to the variety of upgrade, the management of the lesion has been a matter of discussion. While some centres preferred a more surgical approach with a surgical excision of the lesions, others, due to the low risk of malignancy, preferred the clinical and radiological follow up, especially in those lesions without atypia [6].

In 2018 NHS Breast Screening Programme published guidelines for the management of B3 lesion in the Screening Programme, which were in line with the already published International Guidelines. In those guidelines there was a clear differentiation in the management of the papillary lesions without atypia and those with atypia. The lesions presenting with atypia were surgically managed with surgical excision to evaluate the extent of the continuity of the atypia and for better differentiation of DCIS. The pathway changed radically for those papillary lesions with no atypia. The guidelines proposed after an initial diagnosis of papillary lesions with no atypia a subsequent excision of the lesion with a vacuum assisted excision. The rational for this proposal was that VAE is an alternative, cost effective, non-surgical technique which allows a further evaluation of a larger amount of tissue. The aim of the VAE is to provide a wider sampling of the lesion and the surrounding area to exclude associated malignancy. The easiest approach is to guide the VAE with an ultrasound, as it shows in real time how the lesion is been sampled and 
allows evaluation of residual lesions. The aim of the VAE is not to remove entirely the lesion but to remove at least 4 grams of tissue (or 12 cores of $7 \mathrm{G}$ biopsy) or remove at least one third of the lesion (considered as representative sampling). Small lesions of less than $15 \mathrm{~mm}$ are easily removed, while some bigger lesions cannot be removed completely. After the VAE, if there is no associated atypia, the lesions were considered benign and therefore the patient was discharged; if atypia or malignancy were present, then the lesions would be surgically removed.

In our sample, no correlation was found between the size of the lesions or the ultrasound appearances and the upgrade to atypia [7].

The VAE is an easy and cost effective technique, well tolerated by the patients, with no major complications. Some units have reported bleeding or dimpling in a small percentage of cases. The patients can avoid unwanted complications of surgery, as anaesthetic or scarring.

Our unit embraced the guidelines published by NHSBSP in August 2018. During this time we have strictly adhered to the protocol and have not had any upgrades from papillary lesions without atypia to malignancy. The procedure is easy and quick to perform with no complications reported. The patients tolerate the procedure easily with no concerns.

Some of the challenges faced were that in small lesions, less than 3 cores were needed to excise completely the lesion. In these cases, double the number of cores was taken as a precaution. A second challenge was close proximity to the nipple or the skin, as per the complications described in the literature. We have not had any dimpling or nipple retraction (including in the superficial lesions).

Some of the limitations of our study are the small number of patients included and the lack of long term follow up. Notwithstanding, performing VAE in papillary lesion with no atypia is a quick procedure, well tolerated by patients and avoids surgery (avoiding costs and emotional distress) and has efficiently ruled out malignancy in all our cases.

\section{Conclusion}

Papillary lesions with no atypia have a low potential of malignancy but are considered B3 lesions. They can be easily excised with an US guided vacuum assisted excision. Vacuum assisted excision is an easy procedure and well tolerated by the patient. It may be considered as an alternative to treat papillary lesions with no atypia, as per the low percentage of complications.

\section{Additional files}

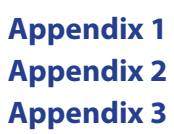

\section{Competing interests}

The authors declare that they have no competing interests.

Authors' contributions

\begin{tabular}{|l|c|c|c|c|c|}
\hline Authors' contributions & TPA & JJ & AK & RG & MM \\
\hline Research concept and design & $\checkmark$ & -- & -- & -- & -- \\
\hline Collection and/or assembly of data & $\checkmark$ & -- & -- & $\checkmark$ & -- \\
\hline Data analysis and interpretation & $\checkmark$ & $\checkmark$ & -- & -- & -- \\
\hline Writing the article & $\checkmark$ & $\checkmark$ & -- & -- & -- \\
\hline Critical revision of the article & $\checkmark$ & $\checkmark$ & $\checkmark$ & -- & $\checkmark$ \\
\hline Final approval of article & $\checkmark$ & $\checkmark$ & -- & -- & -- \\
\hline Statistical analysis & $\checkmark$ & -- & -- & -- & -- \\
\hline
\end{tabular}

Publication history

Editor: Wei-Hsiung Yang, Mercer University School of Medicine, USA.

Received: 03-Feb-2020 Final Revised: 28-Feb-2020

Accepted: 03-Mar-2020 Published: 13-Mar-2020

\section{References}

1. Jorns JM. Papillary Lesions of the Breast: A Practical Approach to Diagnosis. Arch Pathol Lab Med. 2016; 140:1052-9. I Article I PubMed

2. Pinder SE, Shaaban A, Deb R, Desai A, Gandhi A, Lee AHS, Pain S, Wilkinson $L$ and Sharma N. NHS Breast Screening multidisciplinary working group guidelines for the diagnosis and management of breast lesions of uncertain malignant potential on core biopsy (B3 lesions). Clin Radiol. 2018; 73:682-692. | Article I PubMed

3. Rageth CJ, O’Flynn EA, Comstock C, Kurtz C, Kubik R, Madjar H, Lepori D, Kampmann G, Mundinger A, Baege A, Decker T, Hosch S, Tausch C, Delaloye JF, Morris E and Varga Z. First International Consensus Conference on lesions of uncertain malignant potential in the breast (B3 lesions). Breast Cancer Res Treat. 2016; 159:203-13. | Article | PubMed Abstract | PubMed FullText

4. Rageth CJ, O'Flynn EAM, Pinker K, Kubik-Huch RA, Mundinger A, Decker T, Tausch C, Dammann F, Baltzer PA, Fallenberg EM, Foschini MP, Dellas S, Knauer M, Malhaire C, Sonnenschein M, Boos A, Morris E and Varga Z. Second International Consensus Conference on lesions of uncertain malignant potential in the breast (B3 lesions). Breast Cancer Res Treat. 2019; 174:279-296. | Article | PubMed Abstract | PubMed FullText

5. A. Shaaban and N. Sharma. Management of B3 lesions- Practical issue. Current Breast cancer reports. 2019; 11:83-88.

6. Yu Y, Salisbury E, Gordon-Thomson D, Yang JL and Crowe PJ. Management of papillary lesions without atypia of the breast diagnosed on needle biopsy. ANZ J Surg. 2019; 89:524-528. | $\underline{\text { Article | PubMed }}$

7. S. Holley et al. Pathologic Outcomes of non malignant papillary breast lesions diagnosed with imaging-guided core needle biopsy. Radiology. 2012; 265:379-384.

\section{Citation:}

Abos TP, Juttla J, Kumar A, Gardy R and Moreland M. Ultrasound guided vacuum assisted excision of breast papillary lesions with no atypia: Single centre experience after 2018 UK Breast Screening guidelines. Breast Cancer Rep. 2020; 7:1.

http://dx.doi.org/10.7243/2057-1631-7-1 